\title{
ERRATUM
}

A.A.S. 1, 319-343 (2007)

\section{APPLICATION OF A POLYGENIC MODEL OF BREAST AND OVARIAN CANCER TO CRITICAL ILLNESS INSURANCE}

A typographical error in Antoniou et al. (2002) was carried forward into Macdonald \& McIvor (2007), in which paper it affected the numerical results, though not the overall conclusions. The error was in the displayed equation on page 77 of Antoniou et al. (2002); the term $\sigma_{P}$ appeared in the numerator instead of in the denominator. Thus, equation (6) in Macdonald \& McIvor (2007) (using their notation) should read:

$$
R \approx \frac{P}{\sqrt{n / 2}} \sigma_{R}
$$

As a result, the rates of onset of breast cancer given a value $P$ of the polygene (equation (5) in Macdonald \& McIvor (2007)) contain a term $e^{R}$ in which $R$ is understated by a factor $\sigma_{r}^{2}=1.291$. The range of breast cancer risk conferred by the polygene is therefore greater than was shown in Macdonald \& McIvor (2007). It is evident that the corrected results shown here do not contradict their conclusions, in fact they lend them greater emphasis.

The results affected are those shown in Tables 3, 5, 6, 7 and 8, and in Figures 3 and 4 of Macdonald \& McIvor (2007), corrected versions of which follow.

We understand that an erratum correcting the error in Antoniou et al. (2002) will appear in British Journal of Cancer.

\section{REFERENCES}

Antoniou, A.C., Pharoah, P.D.P., McMullan, G., Day, N.E., Stratton, M.R., Peto, J., Ponder, B.J. \& Easton, D.F. (2002). A comprehensive model for familial breast cancer incorporating BRCA1, BRCA2 and other genes. British Journal of Cancer, 86, 76-83.

Macdonald, A.S. \& McIvor, K.R. (2006). Application of a polygenic model of breast and ovarian cancer to critical illness insurance. Annals of Actuarial Science, 1, 319-343. 


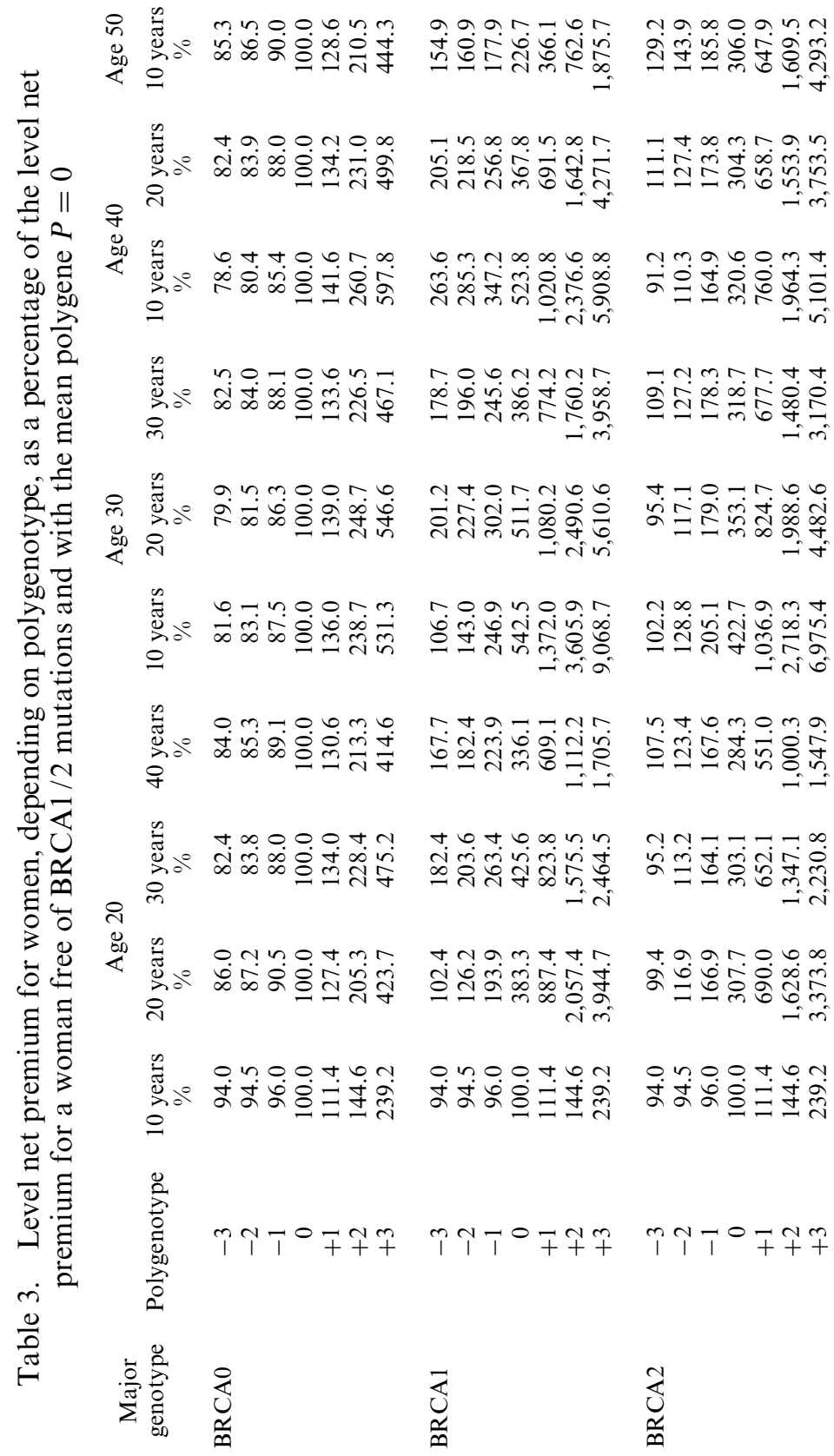




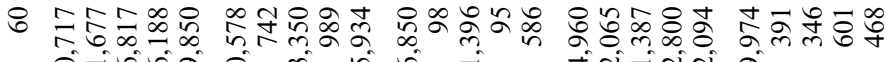
îं -

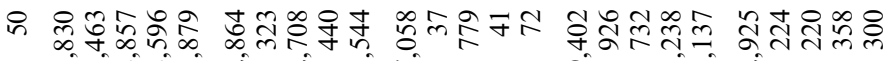

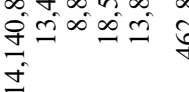

ลूँ

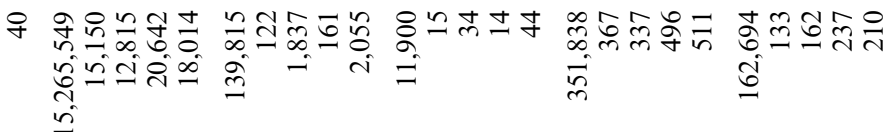

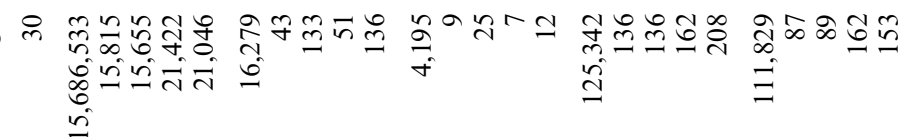

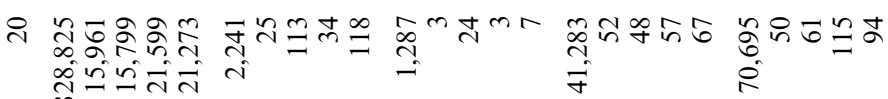

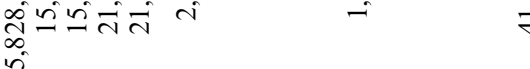

촐

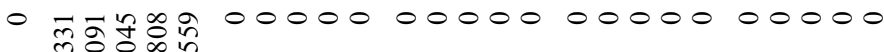

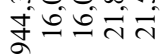

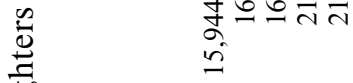

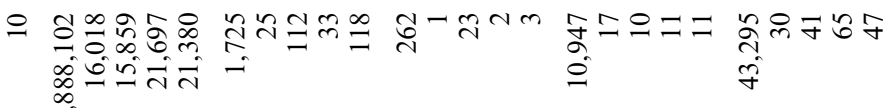
i

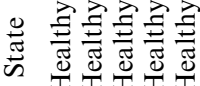
U.

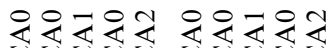
UUU U UUU UU 
Table 6. Numbers of daughters with a family history and given major genotype, in each state in the CI model (see Figure 2), at selected ages

Genotype

$\begin{array}{llllrlrrrr}\text { Family } & \text { Applicant } & \text { State } & 0 & 10 & 20 & 30 & 40 & 50 & 60 \\ \text { BRCA0 } & \text { BRCA0 } & \text { Healthy } & 0 & 0 & 0 & 80 & 5,936 & 48,761 & 35,837 \\ \text { BRCA1 } & \text { BRCA0 } & \text { Healthy } & 0 & 0 & 0 & 1 & 234 & 799 & 666 \\ \text { BRCA1 } & \text { BRCA1 } & \text { Healthy } & 0 & 0 & 0 & 6 & 148 & 427 & 288 \\ \text { BRCA2 } & \text { BRCA0 } & \text { Healthy } & 0 & 0 & 0 & 4 & 175 & 763 & 633 \\ \text { BRCA2 } & \text { BRCA2 } & \text { Healthy } & 0 & 0 & 0 & 3 & 134 & 497 & 244 \\ \text { BRCA0 } & \text { BRCA0 } & \text { BC } & 0 & 0 & 0 & 62 & 6,180 & 65,908 & 74,279 \\ \text { BRCA1 } & \text { BRCA0 } & \text { BC } & 0 & 0 & 0 & 0 & 58 & 233 & 284 \\ \text { BRCA1 } & \text { BRCA1 } & \text { BC } & 0 & 0 & 0 & 1 & 687 & 1,938 & 2,004 \\ \text { BRCA2 } & \text { BRCA0 } & \text { BC } & 0 & 0 & 0 & 0 & 70 & 287 & 357 \\ \text { BRCA2 } & \text { BRCA2 } & \text { BC } & 0 & 0 & 0 & 1 & 573 & 2,049 & 2,237 \\ \text { BRCA0 } & \text { BRCA0 } & \text { OC } & 0 & 0 & 0 & 11 & 278 & 2,869 & 3,342 \\ \text { BRCA1 } & \text { BRCA0 } & \text { OC } & 0 & 0 & 0 & 0 & 4 & 17 & 21 \\ \text { BRCA1 } & \text { BRCA1 } & \text { OC } & 0 & 0 & 0 & 0 & 13 & 322 & 364 \\ \text { BRCA2 } & \text { BRCA0 } & \text { OC } & 0 & 0 & 0 & 0 & 3 & 20 & 22 \\ \text { BRCA2 } & \text { BRCA2 } & \text { OC } & 0 & 0 & 0 & 0 & 9 & 37 & 63 \\ \text { BRCA0 } & \text { BRCA0 } & \text { Other } & 0 & 0 & 0 & 0 & 99 & 2,947 & 6,411 \\ \text { BRCA1 } & \text { BRCA0 } & \text { Other } & 0 & 0 & 0 & 0 & 5 & 55 & 124 \\ \text { BRCA1 } & \text { BRCA1 } & \text { Other } & 0 & 0 & 0 & 0 & 8 & 47 & 70 \\ \text { BRCA2 } & \text { BRCA0 } & \text { Other } & 0 & 0 & 0 & 0 & 5 & 49 & 101 \\ \text { BRCA2 } & \text { BRCA2 } & \text { Other } & 0 & 0 & 0 & 0 & 5 & 28 & 57 \\ \text { BRCA0 } & \text { BRCA0 } & \text { Dead } & 0 & 0 & 0 & 0 & 42 & 767 & 1,383 \\ \text { BRCA1 } & \text { BRCA0 } & \text { Dead } & 0 & 0 & 0 & 0 & 3 & 14 & 23 \\ \text { BRCA1 } & \text { BRCA1 } & \text { Dead } & 0 & 0 & 0 & 0 & 4 & 15 & 23 \\ \text { BRCA2 } & \text { BRCA0 } & \text { Dead } & 0 & 0 & 0 & 0 & 5 & 16 & 22 \\ \text { BRCA2 } & \text { BRCA2 } & \text { Dead } & 0 & 0 & 0 & 0 & 4 & 16 & 26\end{array}$


ㄷ
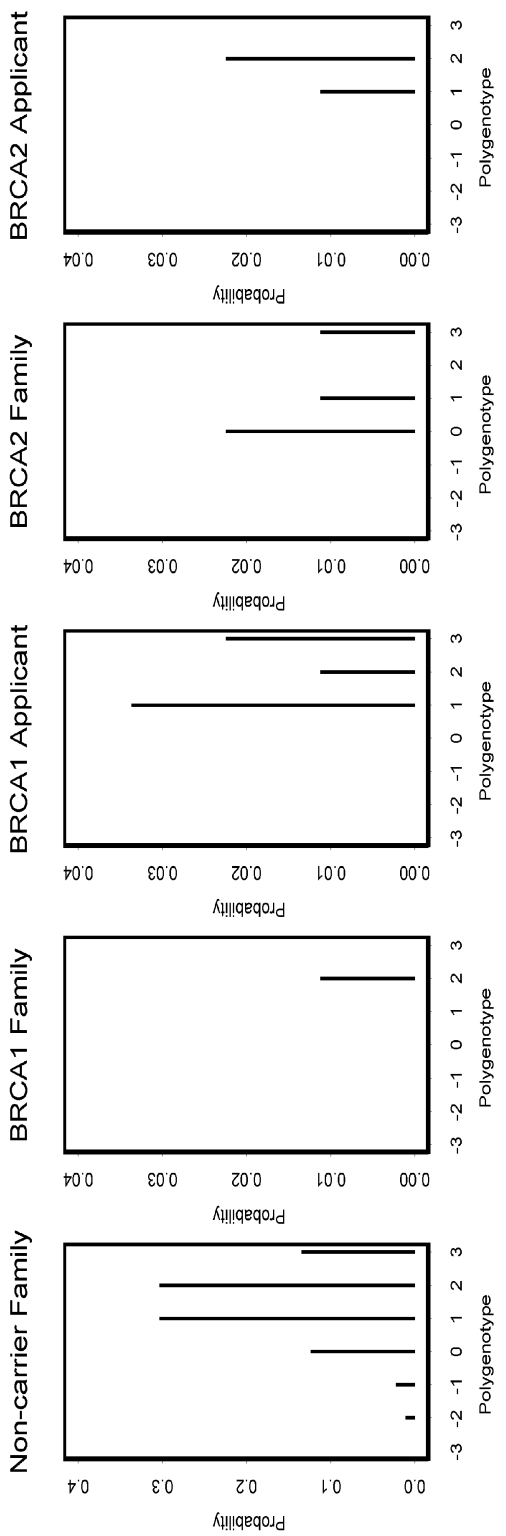

\begin{aligned} &$\hat{N} \\ & \multicolumn{1}{c}{} 0 \\ & 0\end{aligned}$ 疍 คे

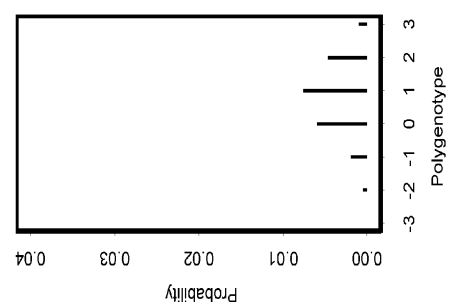

롱

유 공

목

에

음

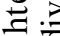

일

ㅋ.

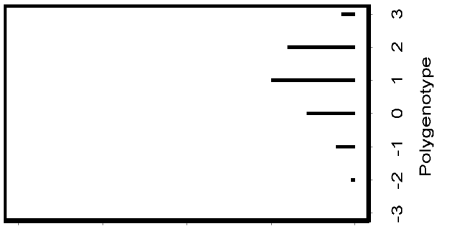

$\lambda$

志.

छ

已

이퓨

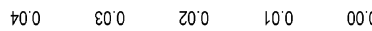

אi!!!geqoud

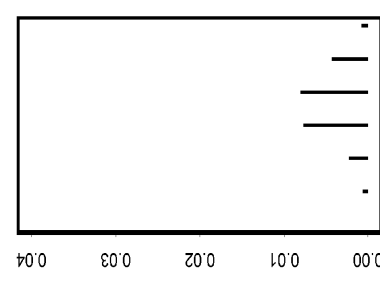

0

8

in

응

ปี $\bar{\Xi}$

का

t)

T

光

글

क $\Xi$

क क

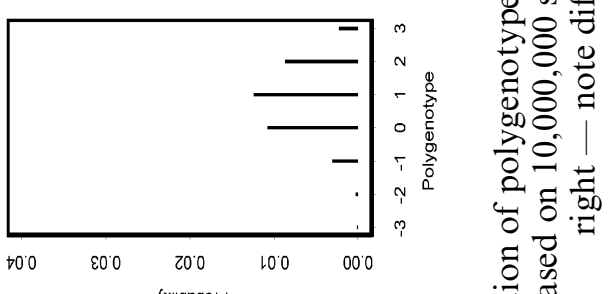

$\because$

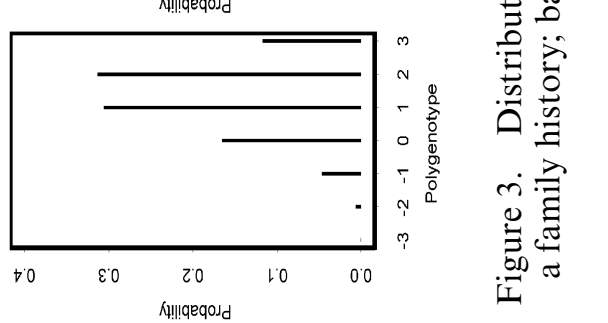



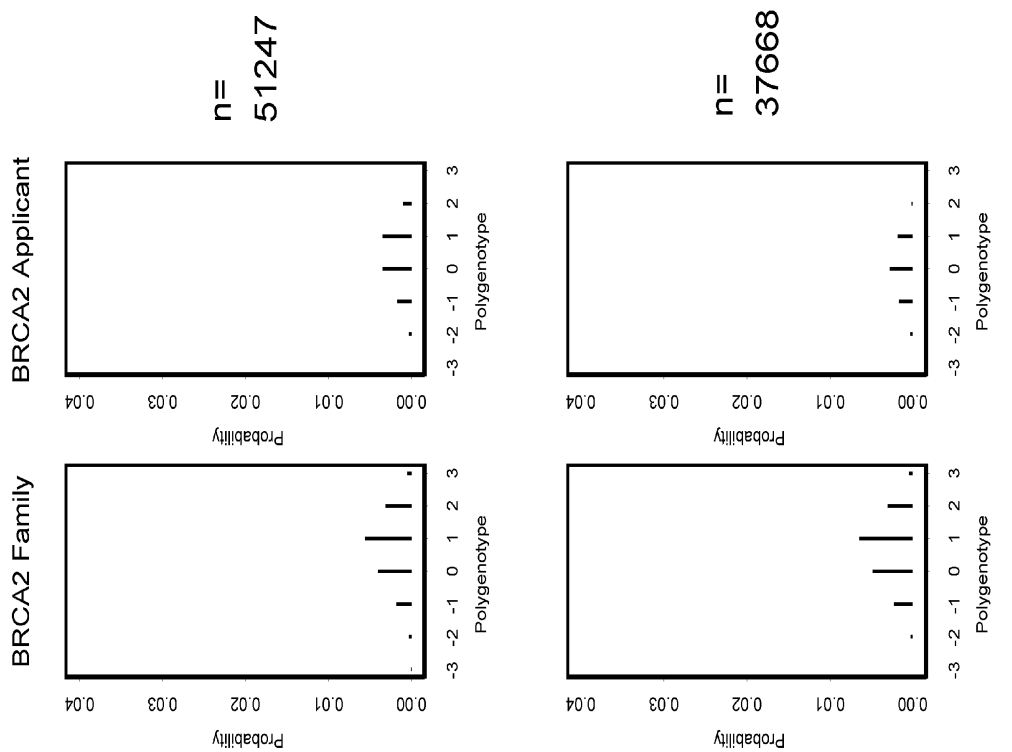

돌

3

ชำ

요

를

융

రㄹ.

कo

금

I :

이름
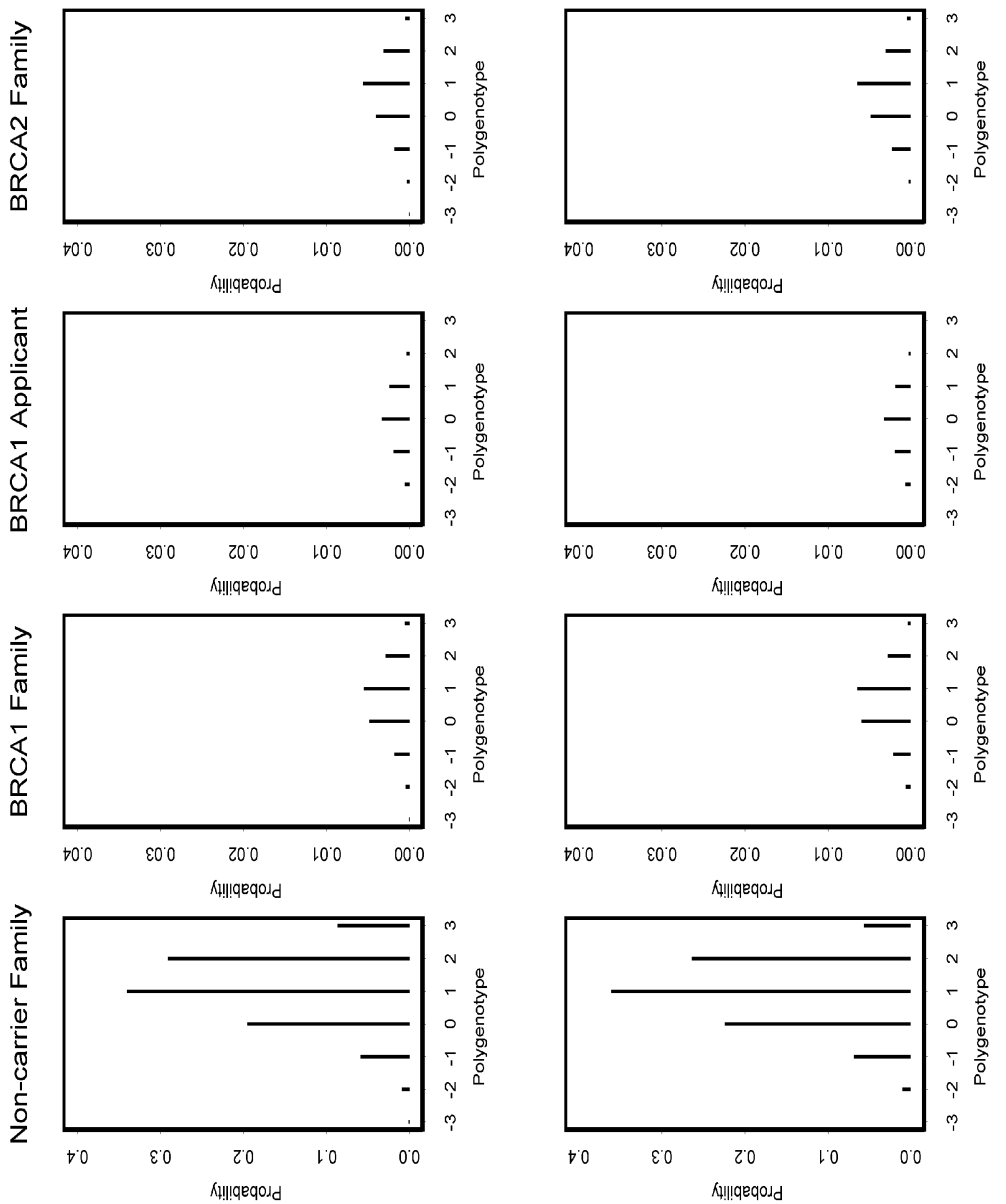

坖范

처

悹司

크

車䲶

흉

ㄴ.

ه

六的先

엉

흘

os

-

氶边

合节

क.

क क

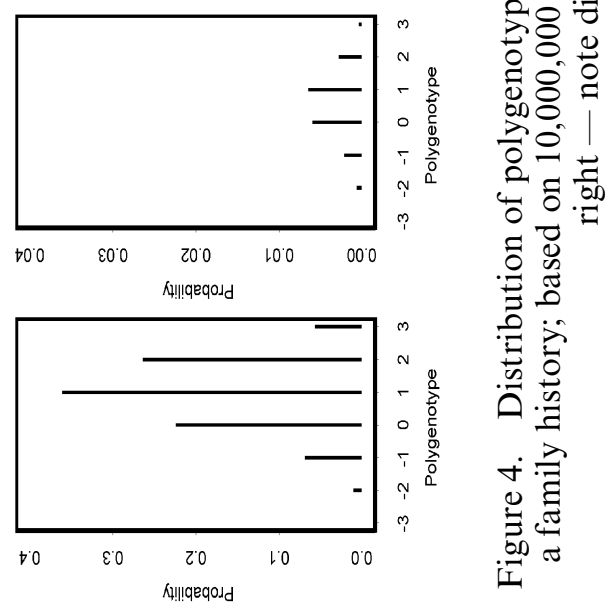


Table 7. Level net premium for females with a family history of $\mathrm{BC}$ or $\mathrm{OC}$, as a percentage of the level net premium for a woman free of BRCA1/2 mutations and with polygenotype $P=0$; the $\mathrm{P}+\mathrm{MG}$ model uses both major gene and polygene probabilities in the weighted average EPVs, while the MG model uses only the major gene probabilities

\begin{tabular}{|c|c|c|c|c|c|c|c|}
\hline \multirow{2}{*}{$\begin{array}{l}\text { Definition of } \\
\text { family history }\end{array}$} & \multirow{2}{*}{$\begin{array}{l}\text { Genetic } \\
\text { model }\end{array}$} & \multicolumn{3}{|c|}{ Age 30} & \multicolumn{2}{|c|}{ Age 40} & Age 50 \\
\hline & & $\begin{array}{c}10 \text { years } \\
\%\end{array}$ & $\begin{array}{c}20 \text { years } \\
\%\end{array}$ & $\begin{array}{c}30 \text { years } \\
\%\end{array}$ & $\begin{array}{c}10 \text { years } \\
\%\end{array}$ & $\begin{array}{c}20 \text { years } \\
\%\end{array}$ & $\begin{array}{c}10 \text { years } \\
\%\end{array}$ \\
\hline 2 affected FDRs & $\begin{array}{c}\mathrm{P}+\mathrm{MG} \\
\mathrm{MG}\end{array}$ & $\begin{array}{l}444.0 \\
137.5\end{array}$ & $\begin{array}{l}341.0 \\
132.0\end{array}$ & $\begin{array}{l}274.7 \\
122.7\end{array}$ & $\begin{array}{l}244.2 \\
112.9\end{array}$ & $\begin{array}{l}207.4 \\
108.9\end{array}$ & $\begin{array}{l}170.6 \\
102.8\end{array}$ \\
\hline 3 affected FDRs & $\begin{array}{c}\mathrm{P}+\mathrm{MG} \\
\mathrm{MG}\end{array}$ & $\begin{array}{l}100.0 \\
100.0\end{array}$ & $\begin{array}{l}100.0 \\
100.0\end{array}$ & $\begin{array}{l}100.0 \\
100.0\end{array}$ & $\begin{array}{l}410.7 \\
148.2\end{array}$ & $\begin{array}{l}314.1 \\
134.3\end{array}$ & $\begin{array}{l}215.6 \\
106.8\end{array}$ \\
\hline 4 affected FDRs & $\begin{array}{c}\mathrm{P}+\mathrm{MG} \\
\mathrm{MG}\end{array}$ & $\begin{array}{l}100.0 \\
100.0\end{array}$ & $\begin{array}{l}100.0 \\
100.0\end{array}$ & $\begin{array}{l}100.0 \\
100.0\end{array}$ & $\begin{array}{l}934.3 \\
207.9\end{array}$ & $\begin{array}{l}637.1 \\
173.2\end{array}$ & $\begin{array}{l}260.8 \\
112.1\end{array}$ \\
\hline
\end{tabular}

Table 8. Level net premium for females with a family history of BC or $\mathrm{OC}$, as a percentage of the standard premium; the polygenic model is compared with the major-gene-only model of Gui et al. (2006); the latter assumed that onset rates of $\mathrm{BC}$ and $\mathrm{OC}$ among $\mathrm{BRCA} 1 / 2$ mutation carriers were either $100 \%$ or $50 \%$ of those estimated, as a rough allowance for ascertainment bias

$\begin{array}{cccccccc}\begin{array}{c}\text { Definition of } \\ \text { family history }\end{array} & \begin{array}{c}\text { Genetic } \\ \text { model }\end{array} & \begin{array}{c}10 \text { years } \\ \%\end{array} & \begin{array}{c}20 \text { years } \\ \%\end{array} & \begin{array}{c}30 \text { years } \\ \%\end{array} & \begin{array}{c}10 \text { years } \\ \%\end{array} & \begin{array}{c}20 \text { years } \\ \%\end{array} & \begin{array}{c}\text { Age } 50 \\ \%\end{array} \\ 2 \text { affected FDRs } & \text { P+MG } & 444.0 & 341.0 & 274.7 & 244.2 & 207.4 & 170.6 \\ & \text { MG } & 137.5 & 132.0 & 122.7 & 112.9 & 108.9 & 102.8 \\ \text { Gui } \text { et al. (2006) } & 100 \% & 330 & 251 & 204 & 208 & 174 & 142 \\ & 50 \% & 217 & 179 & 156 & 154 & 139 & 120\end{array}$

